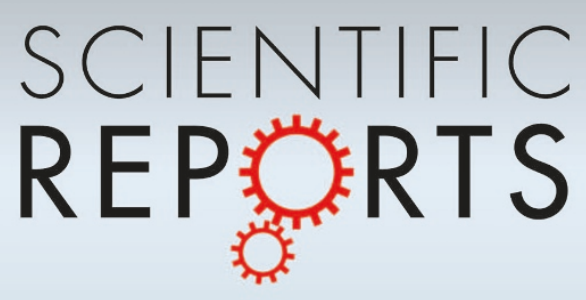

OPEN

SUBJECT AREAS:

PHYSICS

MATERIALS SCIENCE

CONDENSED-MATTER PHYSICS

SUPERCONDUCTING PROPERTIES

AND MATERIALS

Received

12 June 2013

Accepted

15 August 2013

Published

30 August 2013

Correspondence and requests for materials should be addressed to

L.L.S. (llsun@iphy.ac. cn) or Z.X.Z.

(zhxzhao@iphy.ac.cn)

\section{Observation of antiferromagnetic order collapse in the pressurized insulator LaMnPO}

\author{
Jing Guo', J. W. Simonson', Liling Sun', Qi Wu', Peiwen Gao', Chao Zhang', Dachun Gu', \\ Gabriel Kotliar', Meigan Aronson² \& Zhongxian Zhao'
}

'Institute of Physics and Beijing National Laboratory for Condensed Matter Physics, Chinese Academy of Sciences, Beijing 100190, China, ${ }^{2}$ Department of Physics and Astronomy, Stony Brook University, Stony Brook, NY 11794 , USA, ${ }^{3}$ Department of Physics and Astronomy, Rutgers University, Piscataway, NJ 08854, USA.

The emergence of superconductivity in the iron pnictide or cuprate high temperature superconductors usually accompanies the suppression of a long-ranged antiferromagnetic (AFM) order state in a corresponding parent compound by doping or pressurizing. A great deal of effort by doping has been made to find superconductivity in Mn-based compounds, which are thought to bridge the gap between the two families of high temperature superconductors, but the AFM order was not successfully suppressed. Here we report the first observations of the pressure-induced elimination of long-ranged AFM order at $\sim 34 \mathrm{GPa}$ and a crossover from an AFM insulating to an AFM metallic state at $\sim 20 \mathrm{GPa}$ in LaMnPO single crystals that are iso-structural to the $\mathrm{LaFeAsO}$ superconductor by in-situ high pressure resistance and ac susceptibility measurements. These findings are of importance to explore potential superconductivity in Mn-based compounds and to shed new light on the underlying mechanism of high temperature superconductivity.

$\mathrm{T}$

he discovery of iron pnictide superconductor and its extended studies ${ }^{1-7}$ stimulated the investigations on Mn-based superconductors ${ }^{8-14}$. As is found in other Mn-based compounds that are iso-structural to the parent compound of iron pnictide superconductor $\mathrm{LaFeAsO}^{1}$, the physical properties of LaMnPO bear a strong resemblance to those of the parent compounds of cuprate high temperature superconductors ${ }^{3}$. Therefore, it is appropriate to study $\mathrm{LaMnPO}$ with the aim of uncovering the underlying mechanism of high temperature superconductivity and for seeking potential superconductivity. At ambient pressure, LaMnPO is a localized moment AFM insulator with a Néel temperature $\left(\mathrm{T}_{\mathrm{N}}\right)$ about $375 \mathrm{~K}$, and the $\sim 3.2 \mu_{\mathrm{B}}$ per Mn moment align antiferromagnetically in a checkerboard pattern that is stacked along the $c$ axis ${ }^{15,16}$. Like doping studies of $\mathrm{BaMn}_{2} \mathrm{As}_{2}{ }^{17-20}$ and $\mathrm{CaMn}_{2} \mathrm{Sb}_{2}{ }^{21}$, long-ranged AFM order in LaMnPO is also robust upon chemical doping. The substitution of fluorine for oxygen to its solubility limit does not much alter the long-ranged AFM order state in $\mathrm{LaMnP}\left(\mathrm{O}_{1-\mathrm{x}} \mathrm{F}_{\mathrm{x}}\right)$, and no metallization is observed ${ }^{16,22}$. All results that are available so far demonstrate that chemical doping does not have a decisive influence on the long-ranged AFM order in these Mn-based compounds. High-pressure studies on $\mathrm{BaMn}_{2} \mathrm{As}_{2}$ find evidence of metallization ${ }^{23}$, however, no experimental investigation on the variation of the long-range AFM order with pressure has been reported. In this study, we applied high pressure techniques to probe the possible elimination of the long-ranged AFM order and the potential for superconductivity in LaMnPO.

\section{Results}

Figure 1(a) displays the temperature (T) dependence of the electrical resistance $(R)$ of a LaMnPO single crystal under different pressures. Our instrumental restrictions limit the measurement for the $R$ - $T$ curves at pressure lower than $11.7 \mathrm{GPa}$. The low temperature resistances at this pressure show an obvious upturn, which is suppressed remarkably with further increasing pressure. Significantly, a crossover from the insulating behavior to the metallic behavior emerges at pressures of $20.8 \mathrm{GPa}$. By careful inspection of the $R$ - $T$ curves measured at pressures between $20.8 \mathrm{GPa}$ and $25.5 \mathrm{GPa}$, we find a sizable hump with a maximum centered at the temperature T', as indicated by an arrow in Fig. 1b, revealing that there exists an intermediary phase in the compressed LaMnPO. The hump signals that the itinerant and localized electrons coexist and compete with each other, 

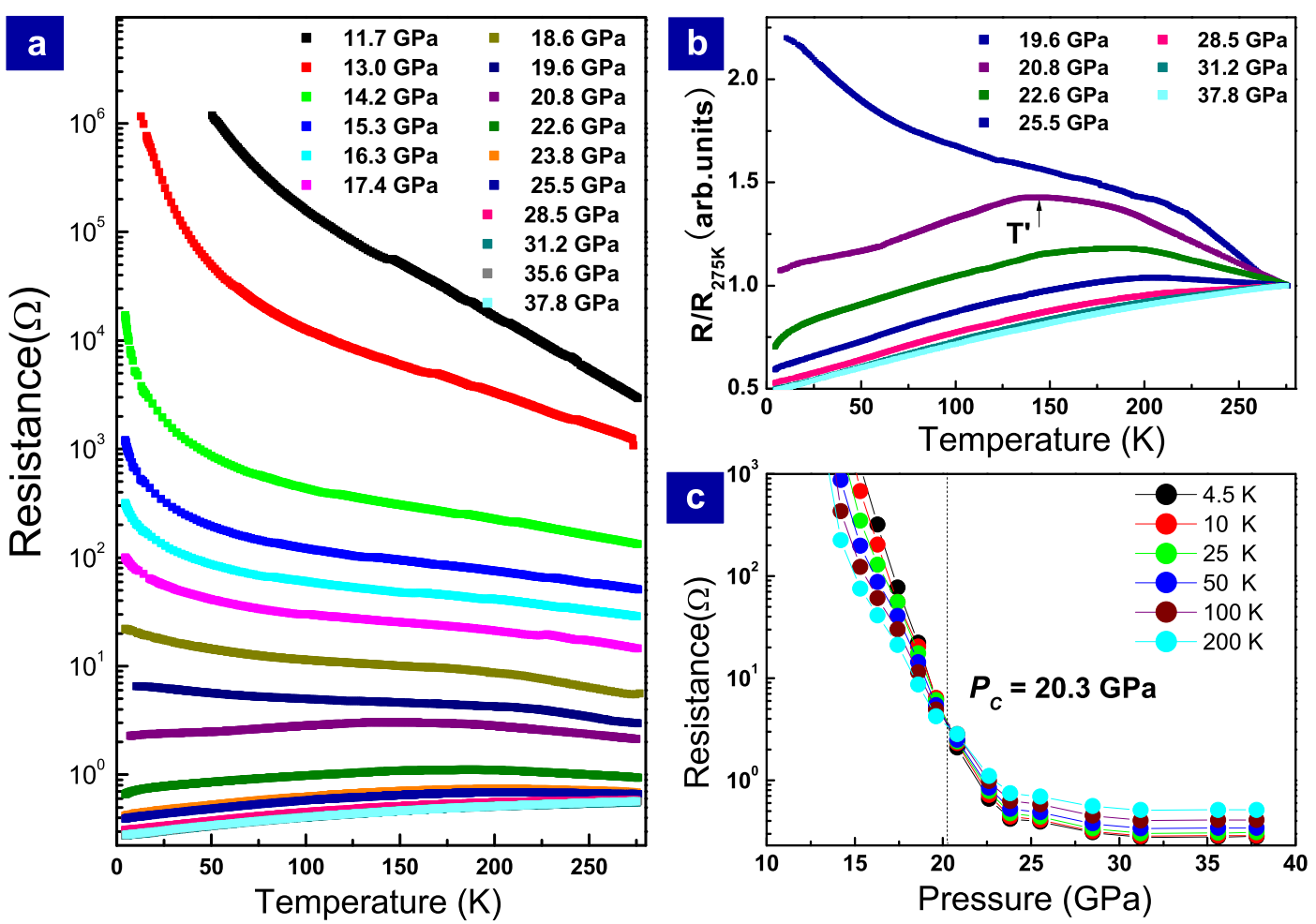

Figure 1 Temperature dependencies of the electrical resistance for the LaMnPO single crystal, measured at different pressures, and resistance as a function of pressure at different fixed temperatures. (a) Resistance-Temperature $(R-T)$ curves measured in the pressure range 11.7-37.8 GPa, displaying a remarkable pressure-induced suppression of the insulating behavior. (b) Selected $R$ - $T$ curves measured in the pressure range 19.6-37.8 GPa, show an intermediary phase featuring a resistance hump. The temperature $\mathrm{T}^{\prime}$ represents the temperature with the maximum resistance, as indicated by the arrow. This hump phenomenon lies in the pressure range 20.8-25.5 GPa, and is completely suppressed at $28.5 \mathrm{GPa}$ and above, where the system enters into a fully metallic state. (c) The pressure dependence of the resistance obtained at different fixed temperatures, exhibiting a critical pressure $\left(\mathrm{P}_{\mathrm{C}}\right)$ of the transition from the insulating state to metallic state in LaMnPO.

suggesting the occurrence of partial electronic delocalization in LaMnPO. The hump disappears when pressure is increased above $25.5 \mathrm{GPa}$.

To determine the critical pressure for the crossover precisely, we plot the pressure dependencies of the resistance measured at different temperatures (Fig. 1c). It is noted that the temperature dependence of the resistance changes its trend at the pressure range between 19.6 and $20.8 \mathrm{GPa}$. Thus, we take the average value (20.3 GPa) as the onset pressure $\mathrm{P}_{\mathrm{C}}$ for the crossover. At pressures above $31.2 \mathrm{GPa}$, the resistance becomes almost linearly proportional to temperature (Fig. 1b), indicating that the system enters a pure metallic state.

Figure $2 \mathrm{a}$ shows the resistance as a function of the reciprocal temperature for the LaMnPO single crystal subjected to different pressures. We found that these Arrhenius plots are linear at high temperatures for pressures as large as $25.5 \mathrm{GPa}$. On basis of the Arrhenius equation, $\rho \sim \exp \left(\varepsilon_{A} / 2 k_{B} T\right)$, we can plot the activation energy for the excitation of charge carriers $\left(\varepsilon_{A}\right)$ as a function of pressure for the sample investigated. As shown in Fig. $2 b, \varepsilon_{\mathrm{A}}$ decreases rapidly with increasing pressure below $15 \mathrm{GPa}$, where the insulating behavior is systematically suppressed (Fig. 1a). It is noted that $\varepsilon_{A}$ remains unchanged with a value of about $60 \mathrm{meV}$ in the pressure range of 16-20 GPa. We attribute this gap to the competition between the itinerant and localized electrons. As pressure is elevated above $20.3 \mathrm{GPa}$, the $\varepsilon_{A}$ continues to decline and the metallicity of the system emerges (inset of Fig. 2b). At pressure above $32 \mathrm{GPa}$, we found that $\varepsilon_{A}$ approaches zero, demonstrating that the gap has collapsed.

It is known from the studies of cuprate and iron pnictide superconductors that the full suppression of long-ranged AFM order is a crucial step to realize superconductivity, and one can ask what the case looks like in LaMnPO. We probed the evolution of the onset AFM transition temperature $\mathrm{T}_{\mathrm{N}}$ with pressure by in-situ high pressure $a c$ susceptibility measurement. Since the $\mathrm{T}_{\mathrm{N}}$ of LaMnPO at ambient pressure is about $375 \mathrm{~K}^{15,16}$ which is out of the measuring range of our instrument, we cannot detect the $\mathrm{T}_{\mathrm{N}}$ at pressures less than $7.3 \mathrm{GPa}$, above the pressure of which the AFM transition becomes apparent as shown in Fig. 3. We can see that the $T_{N}$ shifts to lower temperature with increasing pressure, and then vanishes at pressure close to $34 \mathrm{GPa}$ eventually. The disappearance of the $\mathrm{T}_{\mathrm{N}}$ indicates that pressure drives the system undergoing an AFM-PM transition and all electrons related to the AFM order are delocalized. This is in excellent agreement with our high-pressure resistance data, i.e. at pressures above $32 \mathrm{GPa}$, the system exhibits metallic behavior over the entire temperature range (Fig. 1b). This is the first observation of the collapse of long-ranged AFM order in a Mn-based compound that is iso-structural to the iron pnictide superconductors, an event that was predicted by our previous calculations ${ }^{16}$.

\section{Discussion}

To demonstrate the overall behavior of pressurized LaMnPO clearly, the electronic phase diagram of the LaMnPO is presented in Fig. 4. $\mathrm{T}_{\mathrm{N}}$ is indicated by filled triangles, which is systematically suppressed upon increasing pressure. At $\sim 20 \mathrm{GPa}$, the system enters a mixed state (referred to as the $\mathrm{M}^{\prime}$ phase), in which the insulating (I) and metallic (M) states coexist, therefore the long-ranged AFM order is still retained. The AFM-M' regime that is delineated by the resistance hump and the $\mathrm{T}_{\mathrm{N}}$ lies in the pressure range 20-32 GPa. At pressures close to $34 \mathrm{GPa}$, there is no indication of AFM order, demonstrating 

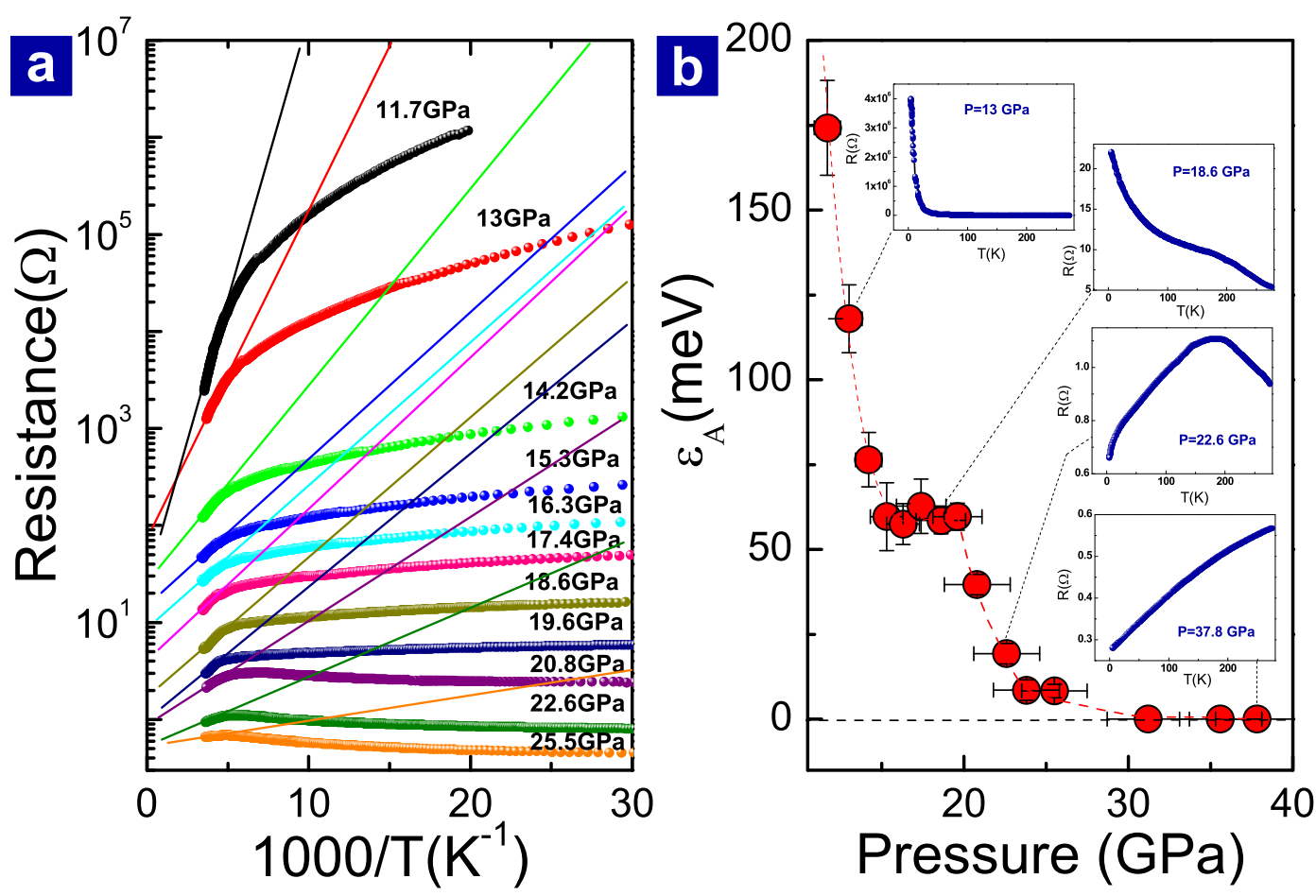

Figure $2 \mid$ Arrhenius plots of the temperature dependence of the resistance at different pressures for the LaMnPO single crystal and its activation energy gap $\varepsilon_{\mathrm{A}}$ as a function of pressure. (a) Resistance as a function of the reciprocal temperature, measured at different pressures. Solid lines are the best high-temperature fits. (b) The pressure dependence of the activation energy gap $\varepsilon_{A}$, which shrinks rapidly with increasing pressure below 15 GPa, remains almost unchanged in the pressure range 15-20 GPa, declines with further increasing pressure, and finally vanishes at a pressure of $32 \mathrm{GPa}$. The insets display the corresponding $R-T$ curves measured at representative pressures.

that the long-ranged AFM order has collapsed and that the localized/ moment-bearing electrons are now fully delocalized. We note that the system becomes fully metallic (Fig. 1b) only at pressures where the long-ranged AFM order at $\mathrm{T}=0$ has collapsed, implying that the insulating behavior of the intermediary phase requires at least some form of AFM short range order. These results, in particular the resistance hump observed at intermediate pressures, are reminiscent of the experimental findings in compressed $\mathrm{A}_{2} \mathrm{Fe}_{4} \mathrm{Se}_{5}(\mathrm{~A}=\mathrm{K}$ or Tl substituted on $\mathrm{Rb})^{24}$. The coexistence of localized and delocalized carriers in these systems $\mathrm{s}^{24-27}$ has been suggested to be the origin of the hump. In the present study, no superconductivity is found in
LaMnPO above the temperature of $1.5 \mathrm{~K}$. However, the observations of the long-ranged AFM order collapse and the novel intermediary phase provide fresh information for exploring potential superconductivity in Mn-based compounds and provide new insight into the underlying mechanism of high temperature superconductivity.

\section{Methods}

The single crystals of LaMnPO were grown by the $\mathrm{NaCl}-\mathrm{KCl}$ eutectic flux method, as described in Ref 22 and 28. Diamond anvil cells (DACs) were used to create high pressure. The anvil diameter is about $300 \mu \mathrm{m}$. High-pressure electrical resistance experiments were carried out in a DAC using the standard four-probe technique. High-pressure alternating current $(a c)$ susceptibility measurements were conducted

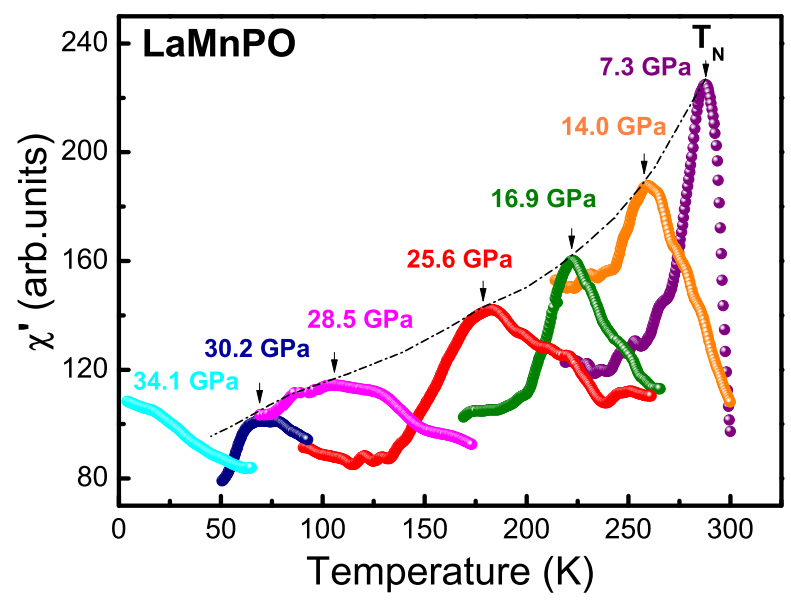

Figure 3 Temperature dependencies of the real part of the alternating current (ac) susceptibility $\chi$ ' measured at different pressures for the LaMnPO single crystal. The arrows indicate maxima in $\chi^{\prime}(\mathrm{T})$ and it marks the onset of AFM transition temperature $\mathrm{T}_{\mathrm{N}}$. The results demonstrate a continuous suppression and elimination of AFM order under pressure. 


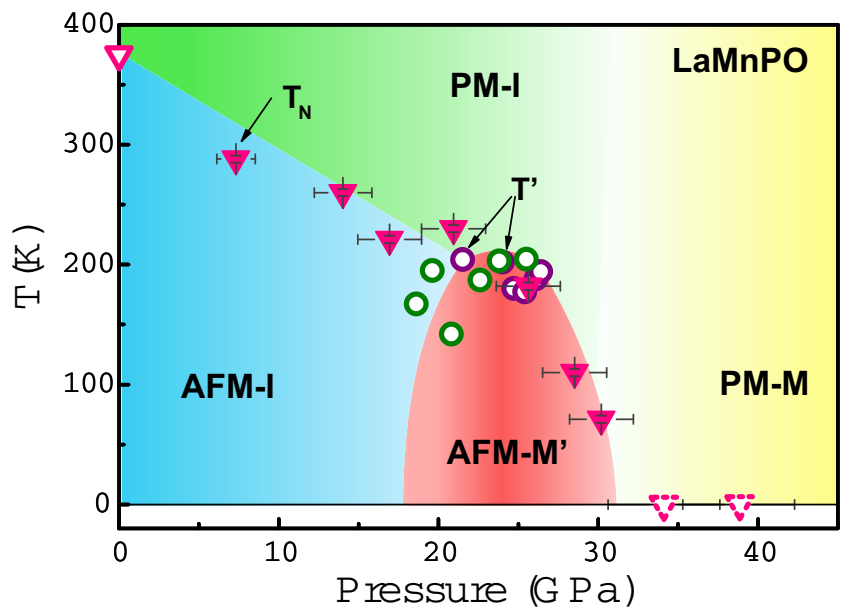

Figure $4 \mid$ The $P$-T electronic phase diagram of LaMnPO. The open triangle represents the AFM transition temperature $\left(\mathrm{T}_{\mathrm{N}}=375 \mathrm{~K}\right)$ at ambient pressure, taken from neutron diffraction measurements ${ }^{15,16}$. The filled triangles correspond to values of $\mathrm{T}_{\mathrm{N}}$ obtained from our high pressure $a c$ susceptibility measurements. The open circles represent temperatures T', where the resistance is at a maximum, taken from different independent runs. There is no indication of AFM order at $\sim 34 \mathrm{GPa}$, as displayed by the dashed open triangles. The acronyms AFM-I and AFM-M' stand for antiferromagnetic insulating and antiferromagnetic mixed state regimes, respectively. PM-I and PM-M represent paramagnetic insulating and paramagnetic metallic states, respectively.

using home-made coils that were wound around a diamond anvil ${ }^{29,30}$. The nonmagnetic rhenium gasket was preindented down to a $50 \mu \mathrm{m}$ thickness for different runs of high-pressure resistance and magnetic measurements. A thin plate sample with dimensions of around $80 \times 80 \times 10 \mu \mathrm{m}$, cleaved from the LaMnPO single crystal, was loaded into the gasket hole in a DAC for the resistance measurements. To detect the $\mathrm{T}_{\mathrm{N}}$ with stronger signal under pressure, several more plates than resistance measurements were loaded into the gasket hole sitting in a different DAC for $a c$ susceptibility measurements. No pressure medium was used in the high-pressure magnetic or resistance measurements. Pressure was determined by ruby fluorescence ${ }^{31}$.

1. Kamihara, Y., Watanabe, T., Hirano, M. \& Hosono, H. Iron-based layered superconductor $\mathrm{La}\left[\mathrm{O}_{1-\mathrm{x}} \mathrm{F}_{\mathrm{x}}\right] \mathrm{FeAs}(\mathrm{x}=0.05-0.12)$ with $\mathrm{T}_{\mathrm{c}}=26 \mathrm{~K}$. J. Am. Chem Soc. 130, 3296-3297 (2008).

2. Zhao, J. et al. Structural and magnetic phase diagram of $\mathrm{CeFeAsO}_{1-\mathrm{x}} \mathrm{F}_{\mathrm{x}}$ and its relation to high-temperature superconductivity. Nat. Mater. 7, 953-959 (2008).

3. Armitage, N. P., Fournier, P. \& Greene, R. L. Progress and perspectives on the electron-doped cuprates. Rev. Mod. Phys. 82, 2421-2487 (2010).

4. Johnston, D. C. The puzzle of high temperature superconductivity in layered iron pnictides and chalcogenides. Adv. Phys. 59, 803-1061 (2010).

5. Paglione, J. \& Greene, R. L. High-temperature superconductivity in iron-based materials. Nat. Phys. 6, 645-658 (2010)

6. Mazin, I. I. Superconductivity gets an iron boost. Nature 464, 183-186 (2010).

7. Colombier, E., Bud'ko, S. L., Ni, N. \& Canfield, P. C. Complete pressuredependent phase diagrams for $\mathrm{SrFe}_{2} \mathrm{As}_{2}$ and $\mathrm{BaFe}_{2} \mathrm{As}_{2}$. Phys. Rev. B 79, 224518 (2009).

8. Singh, Y. et al. Magnetic order in $\mathrm{BaMn}_{2} \mathrm{As}_{2}$ from neutron diffraction measurements. Phys. Rev. B 80, 100403(R) (2009).

9. Singh, Y., Ellern, A. \& Johnston, D. C. Magnetic, transport, and thermal properties of single crystals of the layered arsenide $\mathrm{BaMn}_{2} \mathrm{As}_{2}$. Phys. Rev. B 79, 094519 (2009)

10. An, J. M., Sefat, A. S., Singh, D. J. \& Du, M. H. Electronic structure and magnetism in $\mathrm{BaMn}_{2} \mathrm{As}_{2}$ and $\mathrm{BaMn}_{2} \mathrm{Sb}_{2}$. Phys. Rev. B 79, 075120 (2009).

11. Xu, G. et al. Doping-dependent phase diagram of LaOMAs $(\mathrm{M}=\mathrm{V}-\mathrm{Cu})$ and electron-type superconductivity near ferromagnetic instability. EPL 82, 67002 (2008).

12. Johnston, D. C. et al. Magnetic exchange interactions in $\mathrm{BaMn}_{2} \mathrm{As}_{2}$ : A case study of the $J_{1}-J_{2}-J_{c}$ Heisenberg model. Phys. Rev. B 84, 094445 (2011).

13. Shiomi, Y., Ishiwata, S., Taguchi, Y. \& Tokura, Y. Mott insulator to metal transition in filling-controlled $\mathrm{SmMnAsO}_{1-\mathrm{x}}$. Phys. Rev. B 84, 054519 (2011).

14. Marcinkova, A., Hansen, T. C., Curfs, C., Margadonna, S. \& Bos, J.-W. G. Ndinduced Mn spin-reorientation transition in NdMnAsO. Phys. Rev. B 82, 174438 (2010).

15. Yanagi, H. et al. Antiferromagnetic bipolar semiconductor LaMnPO with ZrCuSiAs-type structure. J. Appl. Phys. 105, 093916 (2009).

16. Simonson, J. W. et al. From antiferromagnetic insulator to correlated metal in pressurized and doped LaMnPO. Proc. Natl. Acad. Sci. U.S.A. 109, E1815-E1819 (2012).

17. Pandey, A., Anand, V. K. \& Johnston, D. C. Large miscibility gap in the $\mathrm{Ba}\left(\mathrm{Mn}_{\mathrm{x}} \mathrm{Fe}_{1-\mathrm{x}}\right)_{2} \mathrm{As}_{2}$ system. Phys. Rev. B 84, 014405 (2011).
18. Pandey, A. et al. $\mathrm{Ba}_{1-\mathrm{x}} \mathrm{K}_{\mathrm{x}} \mathrm{Mn}_{2} \mathrm{As}_{2}$ : An antiferromagnetic local-moment metal. Phys. Rev. Lett. 108, 087005 (2012).

19. Bao, J.-K. et al. Weakly ferromagnetic metallic state in heavily doped $\mathrm{Ba}_{1-\mathrm{x}} \mathrm{K}_{\mathrm{x}} \mathrm{Mn}_{2} \mathrm{As}_{2}$. Phys. Rev. B 85, 144523 (2012).

20. Lamsal, J. et al. Persistence of local-moment antiferromagnetic order in $\mathrm{Ba}_{1-\mathrm{x}} \mathrm{K}_{\mathrm{x}} \mathrm{Mn}_{2} \mathrm{As}_{2}$. Phys. Rev. B 87, 144418 (2013).

21. Simonson, J. W. et al. Magnetic and structural phase diagram of $\mathrm{CaMn}_{2} \mathrm{Sb}_{2}$. Phys. Rev. B 86, 184430 (2012).

22. Simonson, J. W. et al. Gap states in insulating $\mathrm{LaMnPO}_{1-x} \mathrm{~F}_{x}(x=0-0.3)$. Phys Rev. B 84, 165129 (2011).

23. Satya, A. T. et al. Pressure-induced metallization of $\mathrm{BaMn}_{2} \mathrm{As}_{2}$. Phys. Rev. B 84, 180515(R) (2011)

24. Gao, P. W. et al. Pressure-induced insulator-metal transition and the pathway towards superconductivity in alkaline iron selenide compounds. arXiv: 1209.1340.

25. Yi, M. et al. Observation of temperature-induced crossover to an orbital-selective Mott phase in $\mathrm{A}_{\mathrm{x}} \mathrm{Fe}_{2-y} \mathrm{Se}_{2}(\mathrm{~A}=\mathrm{K}, \mathrm{Rb})$ superconductors. Phys. Rev. Lett. 110, 067003 (2013).

26. Yu, R. \& Si, Q. Orbital-selective Mott phase in multiorbital models for alkaline iron selenides $\mathrm{K}_{1-\mathrm{x}} \mathrm{Fe}_{2-\mathrm{y}} \mathrm{Se}_{2}$. Phys. Rev. Lett. 110, 146402 (2013).

27. Yin, Z. P., Haule, K. \& Kotliar, G. Kinetic frustration and the nature of the magnetic and paramagnetic states in iron pnictides and iron chalcogenides. Nat. Mater. 10, 932-935 (2011).

28. Nientiedt, A. T., Jeitschko, W., Pollmeier, P. G. \& Brylak, M. Quaternary equiatomic manganese pnictide oxides $\mathrm{AMnPO}(\mathrm{A}=\mathrm{La}-\mathrm{Nd}, \mathrm{Sm}, \mathrm{Gd}-\mathrm{Dy})$, AMnAsO (A = Y, La-Nd, Sm, Gd-Dy, U), and AMnSbO (A = La-Nd, Sm, Gd) with ZrCuSiAs type structure. Z. Naturforsch., B: J. Chem. Sci. 52, 560-564 (1997).

29. Debessai, M., Matsuoka, T., Hamlin, J. J. \& Schilling, J. S. Pressure-induced superconducting state of europium metal at low temperatures. Phys. Rev. Lett. 102, 197002 (2009)

30. Sun, L. L. et al. Re-emerging superconductivity at 48 kelvin in iron chalcogenides. Nature 483, 67-69 (2012)

31. Mao, H. K., Xu, J. \& Bell, P. M. Calibration of the ruby pressure gauge to $800 \mathrm{kbar}$ under quasi-hydrostatic conditions. J. Geophys. Res. 91, 4673-4676 (1986).

\section{Acknowledgements}

Work in China was supported by the NSCF (Grant No. 11074294), 973 projects (Grant No. 2011CBA00100 and 2010CB923000) and Chinese Academy of Sciences. Research at Stony Brook (J. W. S and M. C. A) and Rutgers (G. K) was supported by the Department of Defense National Security Science and Engineering Faculty Fellowship via the Air Force Office of Scientific Research.

\section{Author contributions}

J.G., P.G., C.Z. and D.G. performed high-pressure resistance and magnetic susceptibility research. J.S. and M.A. grew the single crystals. L.S., M.A., Q.W., G.K., J.G. and Z.Z. wrote the paper. All the authors analyzed the data and discussed the results. 


\section{Additional information}

Competing financial interests: The authors declare no competing financial interests.

How to cite this article: Guo, J. et al. Observation of antiferromagnetic order collapse in the pressurized insulator LaMnPO. Sci. Rep. 3, 2555; DOI:10.1038/srep02555 (2013). cc)(1) (2) This work is licensed under a Creative Commons AttributionBY NC SA NonCommercial-ShareAlike 3.0 Unported license. To view a copy of this license, visit http://creativecommons.org/licenses/by-nc-sa/3.0 